\title{
Post-Traumatic Stress Disorder: Evidence-Based Research for the Third Millennium
}

\author{
Javier Iribarren $^{3}$, Paolo Prolo ${ }^{1,2}$, Negoita Neagos $^{2}$ and Francesco Chiappelli ${ }^{1,2}$ \\ ${ }^{1}$ UCLA School of Dentistry, ${ }^{2}$ Psychoneuroimmunology Group, Inc. and ${ }^{3}$ David Geffen School of \\ Medicine at UCLA, Los Angeles, CA, USA
}

\begin{abstract}
The stress that results from traumatic events precipitates a spectrum of psycho-emotional and physiopathological outcomes. Post-traumatic stress disorder (PTSD) is a psychiatric disorder that results from the experience or witnessing of traumatic or life-threatening events. PTSD has profound psychobiological correlates, which can impair the person's daily life and be life threatening. In light of current events (e.g. extended combat, terrorism, exposure to certain environmental toxins), a sharp rise in patients with PTSD diagnosis is expected in the next decade. PTSD is a serious public health concern, which compels the search for novel paradigms and theoretical models to deepen the understanding of the condition and to develop new and improved modes of treatment intervention. We review the current knowledge of PTSD and introduce the role of allostasis as a new perspective in fundamental PTSD research. We discuss the domain of evidence-based research in medicine, particularly in the context of complementary medical intervention for patients with PTSD. We present arguments in support of the notion that the future of clinical and translational research in PTSD lies in the systematic evaluation of the research evidence in treatment intervention in order to insure the most effective and efficacious treatment for the benefit of the patient.
\end{abstract}

Keywords: post-traumatic stress syndrome - allostasis - evidence-based research - complementary medicine

\section{Introduction}

\section{Post-Traumatic Stress Disorders}

The twenty-first century rose in a ray of hope. The belief was commonly held that an age of worldwide prosperity was beginning with the new millennium. Only a few years ago, people spoke of peace. Today, the general trend in many populations across the globe is fear and anxiety about self and neighbor. Socio-political events have cast a shadow of uneasiness about one's own security and that of significant others at a personal as well as a societal level. (Case in point is Greg, a businessman from Southern California, who happened to be on a business trip in New York city scheduled for September 10-12,

For reprints and all correspondence: Francesco Chiappelli, UCLA School of Dentistry, CHS 63-090, Los Angeles, CA 90095-1668, USA. Tel: +1-310794-6625; Fax: +1-310-794-7109; E-mail: chiappelli@dent.ucla.edu
2001. Following the 9/11 attack, which he barely escaped, he immediately attempted to contact his family in the Southland and to leave New York city. He was on the first plane out: but the plane never took off, instead it was boarded by the New York city SWAT team who, at gun point, arrested a passenger seated four seats in front of Greg's. Greg then drove at night to Philadelphia, where he was eventually able to board a plane and return to his anxious family. To this day, Greg does not fly as often as before, is reticent to fly to the east coast and will not return to do business in New York city. His Type II diabetes has considerably worsened.)

Traumatic events are profoundly stressful. The stress that results from traumatic events precipitates a spectrum of psycho-emotional and physiopathological outcomes. In its gravest form, this response is diagnosed as a psychiatric disorder consequential to the experience of traumatic events.

Post-traumatic stress disorder, or PTSD, is the psychiatric disorder that can result from the experience or witnessing of

(C) The Author (2005). Published by Oxford University Press. All rights reserved.

The online version of this article has been published under an open access model. Users are entitled to use, reproduce, disseminate, or display the open access version of this article for non-commercial purposes provided that: the original authorship is properly and fully attributed; the Journal and Oxford University Press are attributed as the original place of publication with the correct citation details given; if an article is subsequently reproduced or disseminated not in its entirety but only in part or as a derivative work this must be clearly indicated. For commercial re-use, please contact journals.permissions@oxfordjournals.org 
traumatic or life-threatening events such as terrorist attack, violent crime and abuse, military combat, natural disasters, serious accidents or violent personal assaults. Exposure to environmental toxins (e.g. Agent orange, electromagnetic radiation) may result in immune symptoms akin to PTSD in many susceptible patients $(1,2)$.

Subjects with PTSD often relive the experience through nightmares and flashbacks. They report difficulty in sleeping. Their behavior becomes increasingly detached or estranged and is frequently aggravated by related disorders such as depression, substance abuse and problems of memory and cognition. The disorder soon leads to impairment of the ability to function in social or family life, which more often than not results in occupational instability, marital problems and divorces, family discord and difficulties in parenting. The disorder can be severe enough and last long enough to impair the person's daily life and, in the extreme, lead the patient to suicidal tendencies. PTSD is marked by clear biological changes, in addition to the psychological symptoms noted above, and is consequently complicated by a variety of other problems of physical and mental health.

\section{PTSD—A Brief History}

Whereas the terminology of PTSD arose relatively soon following the Vietnam conflict, the observation that traumatic events can lead to this plethora of psychobiological manifestations is not new. During the Civil War, a PTSD-like disorder was referred to as the 'Da Costa's Syndrome' (3), from the American internist Jacob Mendez Da Costa (1833-1900; Civil War duty: military hospital in Philadelphia).

The syndrome was first described by ABR Myers (18381921) in 1870 as combining effort fatigue, dyspnea, a sighing respiration, palpitation, sweating, tremor, an aching sensation in the left pericardium, utter fatigue, an exaggeration of symptoms upon efforts and occasionally complete syncope. It was noted that the syndrome resembled more closely an abandonment to emotion and fear, rather than the 'effort' that normal subjects engage to overcome challenges (4). This classic observation pertains to what we now know of allostasis, as we discuss below. Da Costa reported in 1871 that the disorder is most commonly seen in soldiers during time of stress, especially when fear was involved (3). The syndrome became increasingly observed during the Civil War and during World War I.

\section{PTSD in the US Population Today}

The National Center for PTSD (US Department of Veterans Affairs) made public estimates that whereas the lifetime prevalence of PTSD in the US population was $5 \%$ in men and $10 \%$ in women in the mid-to-late 1990s, the prevalence of PTSD among Vietnam veterans at this same time was at $15.2 \%$. About $30 \%$ of the men and women who have spent time in more recent war zones experience PTSD.

Whereas the onset and progression of PTSD is characteristic for every individual subject, data suggest that most people who are exposed to a traumatic, stressful event will exhibit early symptoms of PTSD in the days and weeks following exposure. Available data from the National Center for PTSD suggest that $\sim 8 \%$ of men and $20 \%$ of women go on to develop PTSD and $\sim 30 \%$ of these individuals develop a chronic form that persists throughout their lifetimes. Complex PTSD, which is also referred to as 'disorder of extreme stress', results from exposure to prolonged traumatic circumstances, such as the year-on end threat of insurgent attacks among our military personnel currently in active deployment.

The National Center for PTSD also estimates that under normal and usual socio-political conditions $8 \%$ of the US population will experience PTSD at some point in their lives, with women $(10.4 \%)$ twice as likely as men (5\%) to develop PTSD. At the beginning of the millennium, it was estimated that 5-6 million US adults suffered from PTSD. Because of the traumatic developments of recent years, and of ongoing turmoil worldwide, it is possible and even probable that the incidence of PTSD will sharply increase within the next decade and that it may become one among the most significant public health concerns of this new century. This threat is all the more serious considering the fact that PTSD symptoms seldom disappear completely; recovery from PTSD is a lengthy, ongoing, gradual and costly process, which is often hampered by continuing reaction to memories. Treatment usually aims at reducing reactions and to diminishing the acuity of the reactions. Treatments also seek to increase the subject's ability to manage trauma-related emotions and to greater confidence in coping abilities.

\section{Focus of this Review}

This work discusses our current understanding about PTSD. It explores current developments in stress research and discusses its applications and implication to the complex psychobiological prognosis of PTSD. The work concludes by presenting a view into the future of PTSD treatment from the perspective of evidence-based medicine, which many regard as the breakopen research of the next decades-systematic and critical research on research to establish and determine what is the best available evidence for treatment for the patients. Indeed, this will be particularly true in the case of subjects with PTSD, if the austere predictions of a sharp rise in prevalence consequential to most recent terrorist and war events worldwide that involve US soldiers and civilians prove true.

\section{Current Views on PTSD}

\section{Assessment}

There are different psychiatric rating instruments and scales that can be used to assess adult PTSD. Some are part of comprehensive diagnostic manuals or instruments: DSM-IV TR (diagnostic criteria for 309.81 PTSD) (5); ICD-10 (F43.1 PTSD, from the International Statistical Classification of Diseases and Related Health Problems, 10th revision); the 
PTSD module, within the Structured Clinical Interview for DSM-IV (6) or the PTSD Keane scale (PK scale) (7), within the Minnesota Multiphasic Personality Inventory-2 (MMPI-2).

Some are designed as either self-reports or as clinicianadministered instruments specifically assessing adult PTSD: Davidson Trauma Scale (8); Distressing Event Questionnaire (9); Impact of Event Scale-Revised (10); Trauma Symptom Checklist-40 (11); PTSD Checklist-Civilian Version (12); Revised Civilian Mississippi Scale for PTSD (13); the Posttraumatic Stress Diagnostic Scale (14); Trauma Symptom Inventory (11); Los Angeles Symptom Checklist (15) or the Clinician-Administered PTSD Scale (CAPS) (16).

The underlying phenomena of PTSD are probably centrally mediated. Case in point is a study targeting women with early childhood abuse-related PTSD that found correlates of the emotional Stroop (17). Subjects with and without PTSD were compared. Both groups underwent PET scanning while performing in the color and emotional Stroop tasks and control condition. The control condition involved naming the color of rows of XXs (red, blue, green and yellow). The active color condition involved naming the color of color words (again with the same four colors), while the semantic context of the word was incongruous with the color. The active emotional condition involved naming the color (again the same four colors) of emotionally charged words (rape, bruise, weapon, and stench). These words have been shown to produce emotional arousal (18). The study examined the effectiveness of the Stroop task as a probe of anterior cingulate function in PTSD, because of the role of the anterior cingulate and medial prefrontal cortex in stress response and emotional regulation. After comparing it with the color Stroop, the emotional Stroop displayed significantly decreased blood flow among the PTSD subjects in the anterior cingulate. Performance in the color Stroop task produced a non-specific activation of the anterior cingulate in both the PTSD and non-PTSD abused women. However, the emotional Stroop produced a relatively lower level blood flow response of anterior cingulate among PTSD abused women. These observations may indicate that PTSD anterior cingulate dysfunction is specific to the neural circuitry of the processing of emotional stimuli. Shin et al. (19) confirmed a relative decrease in blood flow in anterior cingulate activation in combat-related PTSD and also displayed a decreased blood flow for the emotional (but not color) Stroop. Taken together, these findings indicate that PTSD may have a neural component, which could significantly alter psychoneuroendocrine-immune regulation, as discussed below.

\section{PTSD Assessment in the Military}

Certain scales have been developed that specifically target military personnel.

(i) PTSD Checklist-Military Version (12).

(ii) The Mississippi Scale for Combat-Related PTSD (M-PTSD), specifically a screening and diagnostic instrument for combat-related PTSD (20), which validated as well for treatment seeking (21) and community samples (22).

(iii) The Combat Exposure Scale measures the level of war time stress of veterans, an instrument with strong internal consistency $(\alpha=0.85)$ as well as a high test-retest reliability $(r=0.97)(23)$.

(iv) The PK scale, a subscale of the MMPI-2, whose items were selected based on their ability to differentiate among veterans diagnosed with PTSD and those who were not. This scale has strong reliability $(\alpha=0.95)$ and good test-retest reliability ( $r=0.94)$ (7).

(v) The SCID PTSD module is frequently used to assess presence of PTSD among veterans as well $(24,25)$.

(vi) Additional scales have been used to target assessment of PTSD among veterans, including the M-PTSD (26-29), the PK scale $(30,31)$ or the CAPS $(29,32)$.

The prevalence of PTSD diagnosis varies depending on the assessment method. One study compared three measures of PTSD among American and Korean War prisoners of war (POWs). It compared an unstructured self-report interview measure, the M-PTSD and the DSM-III-R SCID instrument. The data showed that partially unstructured interviews and the M-PTSD yielded PTSD prevalence rates of 31 and 33\%, respectively, which were significantly higher than the rate of $26 \%$ yielded by the SCID. Both the unstructured clinical interview and the M-PTSD had equal accuracy, consistently disagreeing with the SCID from 7 to $15 \%$ of assessed cases (33).

Such differences in rates, depending on the assessment instrument may hold significance. According to the study (33) there may be different explanations; self-report instruments like the M-PTSD do not reflect DSM criteria as comprehensibly as the SCID. Symptoms may differ in both intensity and kind among older and younger prisoners of war. In the paradoxical side, it is possible for an individual to be diagnosed with PTSD while reporting minimal stress levels; in fact, subjective stress can be seen as a confounding factor that can have an influence on diagnosis (34).

A PTSD-negative clinical interview occurring simultaneously with a PTSD confirmation of PTSD (or also with a moderate-to-low M-PTSD score) may be indicative of chronic, but stable, PTSD. Such chronic and stable PTSD may not be clinically relevant and may not require focused intervention. They recommend to measure symptom intensity with such instruments as the CAPS (16). Such an approach could decrease PTSD-positive diagnoses among subjects with low levels of distress (33).

\section{Allostasis and PTSD}

\section{Allostasis and the Response to Stress}

Allostasis refers to the psychobiological regulatory process that brings about stability through change of state consequential to stress. Psycho-emotional stress can be defined as a perceived lack, or loss of fit of one's perceived abilities and the demands of one's inner world or the surrounding environment 
(i.e. person/environment fit). Traumatic events that trigger PTSD are perfect examples of such onerous demands that lead to the conscious or unconscious perception on the part of the subject of not being able to cope (35).

The perception of stress is often associated with psychological manifestations of anxiety, irritability and anger, sad and depressed moods, tension and fatigue, and with certain bodily manifestations, including perspiration, blushing or blanching of the face, increased heart beat or decreased blood pressure, and intestinal cramps and discomfort. These signs mirror the spectrum of psychobiological symptoms in PTSD. These manifestations are generally associated with the nature of the stress, its duration, chronicity and severity. A group of symptoms, now referred to as the sickness behavior, is also noted that is associated with clinically relevant changes in the balance between the psychoneuroendocrine and the immune systems (35-37).

It was the renowned nineteenth-century French physiologist, Claude Bernard (1813-1878) who first proposed that defense of the internal milieu (le milieu intérieur, 1856) is a fundamental feature of physiological regulation in mammalian systems, whence the phrase 'homeostasis' was coined. By the early 1930s, Walter Cannon (1871-1945) proposed that organisms engage in a dynamic process of adjustment of the physiological balance of the internal milieu in response to changing environmental conditions. Hans Selye (1907-1982) established the cardinal points of the 'Generalized Stress Response' in his demonstration of concerted physiological responses to stressful challenges.

Stress alters the regulation of both the sympathetic and the parasympathetic branches of the autonomic nervous system, with consequential alterations in hypothalamic control of the endocrine response controlled by the pituitary gland. Autonomic activation and the elevation of hormones, including those produced by the hypothalamic-pituitary-adrenal axis, play a pivotal role in regulating cell-mediated immune surveillance mechanisms, including the production of cytokines that control inflammatory and healing events $(35,36)$. In brief, the perception of stress leads to a significant load upon physiological regulation, including circadian regulation, sleep and psychoneuroendocrine-immune interaction.

In brief, stress is profound alterations in the cross-regulation and interaction of the hormonal-immune regulatory axis. The experience of stress, as well as that of traumatic events and the anxiety-laden recollections thereof, produce a primary endocrine response, which involves the release of glucocorticoids (GCs). GCs regulate cellular immune activity in vivo systemically and locally. They block the production of proinflammatory cytokines (e.g. interleukin[IL]-1 $\beta$ IL-6) and TH1 cytokines (e.g. IL-2) at the molecular level in vitro and in vivo, but may have little effects upon $\mathrm{TH} 2$ cytokines (e.g. IL-4). The net effect of challenging immune cells with GC is to impair immune $\mathrm{T}$ cell activation and proliferation, while maintaining antibody production. The secretion of GC by the adrenal cortex is under the control of the anterior pituitary adrenocorticotropin hormone (ACTH). Immune challenges release pro-inflammatory cytokines (e.g. IL-1 $\beta$, IL-6), which induce hypothalamic secretion of the ACTH inducing factor corticotropin releasing factor (CRF) in animal and in human subjects. Stressful stimuli also lead to the significant activation of the sympathetic nervous system and a rise in the levels of pro-inflammatory cytokines (i.e. IL-1 $\beta$ and IL-6). It follows that the consequences of stress are not uniform. The psychopathological and the physiopathological impacts of stress may be significantly greater in certain people, compared with those of others. The impact of stress is dynamic and multifaceted and the same person may exhibit a variety of manifestations of the psychoneuroendocrine-immune stress response with varying degrees of severity at different times. The outcome of stress can be multivalent (35).

\section{Allostasis and Heterostasis}

The term 'heterostasis' arose from stress research to describe the situation where the demands upon the organism exceed its inherent physiological limiting capacity. Sterling and Eyer (38) used the term 'allostasis' to describe the events that involve mind-body systemic regulation to recover from stress, rather than local feedback. Allostatic regulation now signifies the recovery and the maintenance of internal balance and viability amidst changing circumstances consequential to stress. It encompasses a range of behavioral and physiological functions that direct the adaptive function of regulating homeostatic systems in response to challenges (37-39).

The cumulative load of the allostatic process is the allostatic load. The pathological side effects of failed adaptation are the allostatic overload. Allostasis pertains to the psychobiological regulatory system with variable set points. These set points are characterized by individual differences. They are associated with anticipatory behavioral and physiological responses and are vulnerable to physiological overload and breakdown of regulatory capacities $(39,40)$.

Type 1 allostatic load utilizes, as it were, stress responses as a means of self-preservation by developing and establishing temporary or permanent adaptation skills. The organism aims at surviving the perturbation in the best condition possible and at normalizing the normal life cycle. In Type 2 allostatic load, the stressful challenge is excessive, sustained or continued and drives allostasis chronically. An escape response cannot be found. Type I versus type II allostatic responses curiously reiterate Myers' observations that his patients seem to abandon themselves to the emotion and the fear that assailed them, rather than engage in the effort to counter and to overcome the challenge, which normal subjects typically undertook. Future research in PTSD from the perspective of allostasis may reveal a learned helplessness component, which could become key in the development and evaluation of treatment interventions (Fig. 1).

\section{Allostasis and PTSD}

It is clear that stress research and PTSD research are intertwined. Psychobiological manifestations in PTSD and in 
ALLOSTASIS

maintaining stability through change

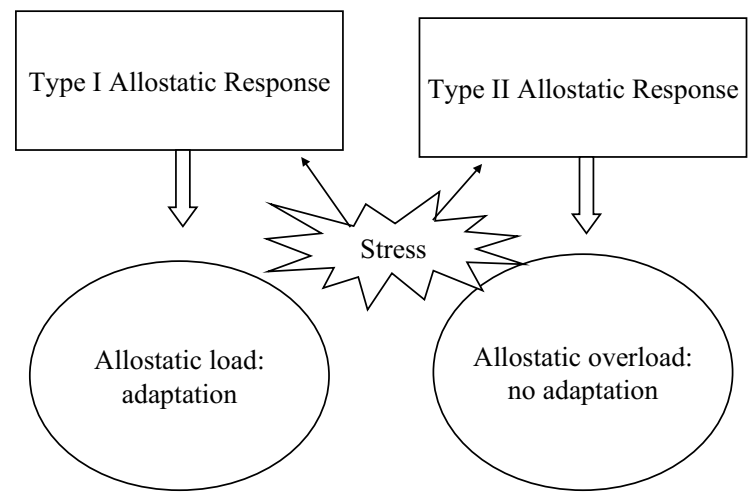

Figure 1. Allostasis refers to the psychobiological regulatory process that brings about stability through change of state consequential to stress. Allostatic regulation describes the recovery and the maintenance of internal balance and viability amidst changing circumstances consequential to stress. It encompasses the Type 1 allostatic load that reflects the utilization by the organism of the range of behavioral and physiological functions that direct the adaptive function of regulating homeostatic systems in response to challenges (i.e. stress response) to develop temporary or permanent adaptation skills by means of self-preservation. Type 1 allostatic responses translate the organism aims at surviving the perturbation in the best condition possible and at normalizing the normal life cycle. By contrast, the Type 2 allostatic responses reflect a load to the organism that is excessive, sustained, or continued, and drives allostasis chronically and that precludes effective escape from the stress. The Type 1 and Type 2 allostatic response dichotomy provides a theoretical model for future research and treatment of PTSD and complex PTSD.

complex PTSD (disorder of extreme stress) evidently pertain to the same domain of mind-body interactions, which are elucidated in psychoneuroimmunology research.

The stress response, more than likely, underlies the psychobiological sequelae of PTSD. The relevance of the field of current research on allostasis to PTSD is all the more evident when one considers that subjects position themselves along a spectrum of allostatic regulation, somewhere between allostasis (i.e. toward regaining physiological balance) and the allostatic overload (i.e. toward physiological collapse and associated potential onset of varied pathologies).

In brief, the recent advances in our understanding of the adaptation of the organism to stressful challenges, the allostatic process, present a new and a rich paradigm for research in the psychobiology of PTSD. Future research must investigate whether or not the dichotomy of Type I and Type II allostatic responses will provide an effective theoretical model for the development of novel and improved modes of intervention to treat PTSD.

\section{PTSD_Paving the Future}

\section{Treatment}

The treatment of PTSD is complex, both in terms of available treatments and the myriad of trauma possibilities that cause it. Properly diagnosing PTSD according to DSM-IV criteria should be the first step, including assessing for co-morbidity. This should be followed by treatments with various degrees of demonstrated efficacy (41).

Historically, it was in the early eighties when research on the treatment efficacy for PTSD began, with multitude of case studies dealing with different kinds of PTSD having been produced since then. Overall, both cognitive behavioral approaches and selective serotonin reuptake inhibitor regimes have been proved to be effective to deal with different kinds of PTSD. At the same time, there is also evidence that other treatment modalities, such as psychodynamic psychotherapy, hypnotherapy, eye movement desensitization and reprocessing can be effective as well; albeit their evidence is derived from less numerous and less well-controlled studies (i.e. open trials or case reports) $(41,42)$. In terms of combined treatments, historically there has not been a systematic effort to address the value of combining medication with psychotherapy and/ or combinations of medications. PTSD intervention is complicated further by the fact that co-morbidities (e.g. substance abuse, over-the-counter medication abuse, psychiatric disorders including major depression) are common. Particularly in situations where co-morbidity exists, a combined approached should be considered.

In addition, there are other considerations affecting the treatment appropriateness:

(i) type of PTSD inducing trauma;

(ii) PTSD chronicity and

(iii) gender, number of times being exposed to trauma and age.

Of interest due to the perilous state of the world (i.e. wars and terrorism) is the issue of the type of PTSD inducing trauma. Combat causes high rates of PTSD and makes it more refractory to treatment than other PTSD-inducing traumas (43). According to experts, combat veterans with PTSD may be less responsive to treatment that other victims of other traumatic exposures $(41,42)$. It is still unclear why combatrelated PTSD is more resistant to treatment than PTSD caused by other traumas. Following is a list of possible reasons:

(i) a great degree of psychopathology presented by patients seeking help at Veterans Administration hospitals;

(ii) isolation from support and help upon returning home and

(iii) potential for secondary gain, such as disability benefits (42).

Combat-caused PTSD is often associated with other psychiatric disorders, including depression, anxiety, mood disorders and substance abuse disorders (22). More specifically, 57$62 \%$ of Croatian Balkan war veterans diagnosed with PTSD also met co-morbid diagnoses criteria (44), with the most common being depression (Muck-Seller et al., 2003), alcohol, drug abuse, phobias, panic disorders and psychosomatic and psychotic disorders (45). In terms of PTSD-associated psychotic symptomatology, between 30 and $40 \%$ of combat-related PTSD subjects may go on to develop psychotic symptomato$\operatorname{logy}(45,46)$. 
It is usually believed that the most effective treatment results are obtained when both PTSD and the other disorder(s) are treated together rather than one after the other. It is becoming increasingly critical to ascertain this position because the prevalence of PTSD and disorder of complex stress is bound to rise sharply in the next decade consequential to the present multinational state of alert and anxiety following ongoing tragic, wanton and widespread terrorism and particularly with respect to combat-related PTSD in present times.

\section{Psychotherapeutic Interventions}

Psychotherapeutic approaches have a long tradition in PTSD treatment, including combat-induced PTSD. Some have more proven efficacy than others. Some of these approaches may be appropriate to address the initial stages of trauma. Psychological debriefing is an intervention given shortly after the occurrence of a traumatic event. The goal is to prevent the subsequent development of negative psychological effects. In fact, psychological debriefing approaches to PTSD can be described as semi-structured interventions aimed at reducing initial psychological stress. Strategies include emotional processing via catharsis, normalization and preparation for future contingencies (47). Gulf War veterans who underwent psychological debriefing showed no significant differences in their scores of two scales measuring PTSD when compared with the control group (48). In general, there is little evidence of psychological debriefing approaches effectively acting to prevent psychopathology, although participants seem to be open to it, which may indicate its usefulness as a rapport builder or as a screening tool. In general however, there is a lack of rigorously conducted research in this area. To this day there is paucity in the data to orient the treatment of combat-related PTSD for veterans (49). The International Consensus Group on Depression and Anxiety supports that exposure psychotherapy is the most appropriate approach for this disorder (41), although this approach does not show a significant influence on PTSD's negative symptomatology, such as avoidance, impaired relationships or anger control (49).

In terms of proven efficacy, cognitive behavior therapy and eye movement desensitization and reprocessing are effective approaches to deal with PTSD (50-54), while other psychotherapeutic approaches (e.g. humanistic or psychodynamic interventions) do not have enough evidence to draw strong conclusions on their utility (42). Cognitive-behavioral psychotherapy encompasses a myriad of approaches (i.e. systematic desensitization, relaxation training, biofeedback, cognitive processing therapy, stress inoculation training, assertiveness training, exposure therapy, combined stress inoculation training and exposure therapy, combined exposure therapy and relaxation training and cognitive therapy). There are empirical studies focusing on PTSD treatment dealing with combatrelated PTSD. Vietnam veterans receiving exposure therapy displayed improvement as evidenced in terms of reducing intrusive combat memories (55), physiological responding, anxiety (56), depression and feelings of alienation, while also promoting increased vigor and skills confidence (57). Exposure therapy, combined with a standard treatment also showed effectiveness with other Vietnam veterans in terms of subject self-report symptoms related to the traumatic experiences, sleep and subjective anxiety responding to trauma stimuli (58).

\section{Pharmacotherapy}

Pharmacotherapy is another approach utilized to deal with PTSD, including combat-induced PTSD. In fact, typically, there is a combination of psychotherapy and medication treatments to treat chronic PTSD (59). In general, the different co-morbidities associated with PTSD play a role in the kinds of pharmacotherapeutic treatments used for its treatment.

Antidepressants and other medications commonly used are tricyclic antidepressants, monoamine oxidase inhibitors, selective serotonin reuptake inhibitors, antianxiety and adrenergic agents and mood stabilizers (60). Sertraline has been found effective to reduce PTSD symptomatology $(61,62)$. In 1999, the United States Food and Drug Administration (FDA) approved sertraline as an appropriate treatment for PTSD. In fact it is the only drug to receive FDA approval to specifically combat PTSD. Sertraline and fluoxetine have produced clinical improvements among PTSD patients in randomized clinical trials (63). Paroxetine, another selective serotonin reuptake inhibitor like sertraline, is also habitually used to treat chronic PTSD (59). Mirtazapine was another successful agent when used in the treatment of PTSD afflicted Korean veterans (64). In addition, Olanzapine and fluphenazine have been successfully used with combat-induced PTSD subjects from the Balkans. Both medicines were successful in ameliorating both PTSD and psychotic symptomatology (43).

Rigorous, well-controlled methods are necessary for conducting studies on the efficacy of PTSD treatments. Well-controlled studies are characterized by the following characteristics:

(i) clearly defined symptoms, as well as inclusion/ exclusion criteria;

(ii) measures used are reliable and valid, with solid psychometric properties;

(iii) utilization of blind evaluators in order to minimize expectancy and demand biases;

(iv) properly trained evaluators to ensure reliability and validity;

(v) the chosen intervention programs are specific, replicable and manualized in order to maximize consistent intervention delivery;

(vi) there is no biased assignment to treatment, which helps maximize that any detected differences and/or similarities are attributable to the treatment technique and not to other causes and

(vii) use of treatment adherence ratings in order to ascertain if intervention parameters were followed (41). 


\section{Research on Research in PTSD: Role of Evidence-Based Research and Complementary Alernative Medicine}

Future clinical research in PTSD requires the stringent, rigorous and systematic approach provided by evidence-based medicine. Evidence-based research in medicine goes beyond the routine narrative literature review. It systematically evaluates the strength of the available evidence and generates a consensus statement of the best available evidence in the form of a systematic review of the available research (Fig. 2).

The future of clinical and translational research in PTSD lies in the systematic evaluation of the research evidence in treatment intervention for the patients. This type of 'research on research' endeavor requires attentive library search of the published materials (e.g. clinical trials) and informal individual communications with the individual researchers and authors.

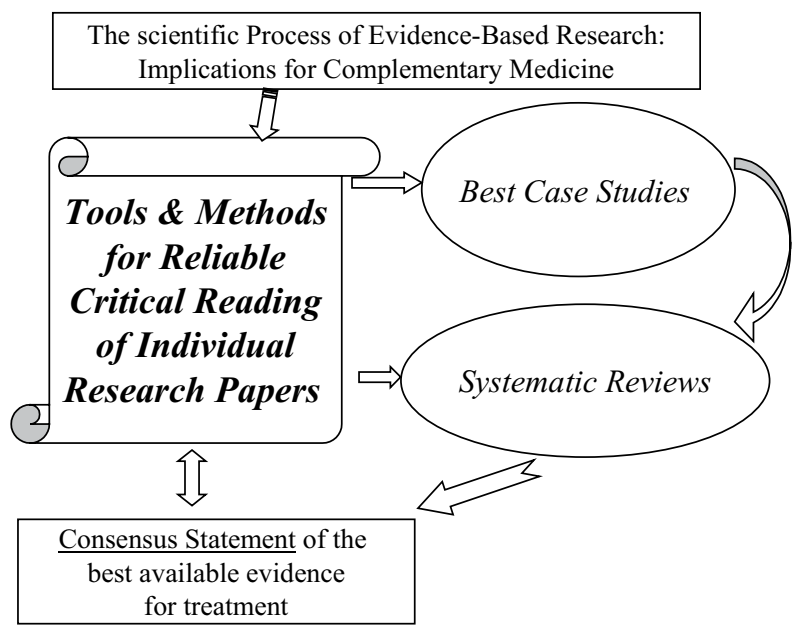

Figure 2. Evidence-based research in medicine follows the 5-step scientific process that includes stating the research question, which in evidence-based research consists of the PIC/PO question (What is the population being examined, e.g. patients with PTSD? What are the interventions being looked at, e.g. conventional treatment versus complementary medicine? Are the interventions being compared or are predictions being drawn, i.e. meta-analysis versus meta-regression approach? What is the outcome of interest, e.g. activities of daily living?). The second step involves methodology, including the sampling of the research literature, and the tools for the critical analysis of the reports. The third step refers to design which usually fall under the acronym CONSORT (i.e. consolidated standards of clinical trials). The fourth step is concerned with the analysis of the data gathered in the evidence-based research process. This commonly entails meta-analytical and meta-regression techniques, as well as individual patient data analysis (e.g. number needed to treat, NNT). Depending upon the tools utilized to evaluate the scientific literature, scores about the completeness and quality of research methodology, design and statistical handling of the findings are generated (SESTA, systematic evaluation of the statistical analysis). These values are analyzed by acceptable sampling statistical protocols to establish whether or not the sample of research reports studied by means of the evidence-based process was statistically acceptable to produce reliable inferences. The last step is a cumulative synthesis, which summarizes the process and the findings. The consensus statement reflects the best available evidence with respect to the stated PIC/PO question. The process is applied to the performance of systematic reviews, which are all-encompassing of the available literature. Best case studies in evidence-based research entail a random performance of the process of evidence-based research with a random sample of the available literature.
The collected evidence is then evaluated for research quality along certain standards [e.g. the consolidated standards of randomized trials (CONSORT)] and by means of validated instruments (e.g. Timmer scale, Jadad scale and Wong scale) (65).

The data from separate reports are pooled, when appropriate, for meta-analysis, meta-regression and individual patient data analyses. The data are analyzed from the perspective of Bayesian modeling in order to interpret data from research in the context of external evidence and judgments (65).

In the context of the treatment of patients with PTSD and co-morbidities, it is important and timely to generate a systematic review of the clinical research evidence for joint and simultaneous treatment of PTSD and the co-morbidities versus a staggered approach. The summative evaluation of the outcome of such a systematic review will generate a consensus statement that will establish whether or not the problem was framed in a clinically relevant manner (e.g. were the patient population, predictor variables and outcome measures clearly identified and relevant to the treatment of PTSD and its co-morbidities within the confines of the research?). The statement must discuss the validity of the process of integration (e.g. were the prospective inclusion and exclusion criteria clearly identified? Was the search comprehensive and explicitly described? Was the validity of the individual studies adequately assessed? Were the process of study selection, searching, assessing validity and data abstraction reliable?). The statement also produces evidence about the rigor of the process by which information was integrated (e.g. were the individual studies sufficiently similar to warrant their combination in an over-arching hypothesis-driven analysis? Are the summary findings representative of the largest and most rigorously performed studies?). The quality, presentation and relevance of the findings must be discussed (e.g. Are the key elements of each study clearly displayed? Is the magnitude of the findings statistically significant? Are the findings homogeneous or heterogeneous? Are sensitivity analyses presented and discussed? Do the findings suggest an overall net benefit for patients with PTSD?). This concerted, systematic and scientific-process driven mode of evaluating current treatment interventions for subjects with PTSD is timely and urgent to insure that the medical establishment will be prepared to handle the fast-approaching wave of PTSD cases in the next decade.

This method-driven approach for the evaluation of clinical data has merit that its product, the consensus statement, must also generate a cost-effectiveness analysis (i.e. a process of decision analysis that incorporates cost) e.g. by a step approach similar as the above method to assess the following:

(i) whether the problem was framed in a clinically relevant manner,

(ii) the validity of integrated information,

(iii) the rigor of process of integration and

(iv) the presentation and quality of the findings.

The relevant findings in this cost-effectiveness analysis are usually expressed as the incremental cost-effectiveness between joint and simultaneous treatment of PTSD and its 
co-morbidities versus a staggered approach. The incremental cost-effectiveness ratio, i.e. the difference in costs between the two strategies divided by the difference in effectiveness between the two strategies, is often presented as well.

The consensus statement evaluates each competitive strategy, usually by means of the Markov model-based decision tree. This approach permits to model events that may occur in the future as a direct effect of treatment or as a side effect. The model produces a decision tree that cycles over fixed intervals in time and incorporates probabilities of occurrence. Even if the difference between the two treatment strategies appears quantitatively small, the Markov model outcome reflects the optimal clinical decision, because it is based on the best possible values for probabilities and utilities incorporated in the tree. The outcome produced by the Markov decision analysis is generally obtained by means of the sensitivity analysis to test the stability over a range of probability estimates and thus reflects the most rational treatment choice (Fig. 3).

The process of evidence-based research in medicine has begun its integration in the domain of PTSD. Rose et al. (66) have established by means of a systematic review of the literature that the early optimism about brief early psychological interventions, including debriefing, is actually unfounded and not supported by the research evidence. These findings confirmed earlier Cochrane-based systematic reviews $(67,68)$. In a separate line of study, systematic reviews established clear support of the research evidence for serotonin reuptake inhibitors as the preferred first line treatment for PTSD, whereas mood stabilizers, atypical neuroleptics, adrenergic agents and newer antidepressants were shown to show promise, but to require further controlled trials to establish their efficacy and efficaciousness $(60,69)$.

The future of clinical and translational research in PTSD also lies in its judicious integration of complementary and alternative medicine (CAM). For instance, whereas PTSD symptoms are common in patients with breast cancer, this symptomatology is more effectively reduced by traditional psychosocial interventions compared with CAM-oriented intervention (70). Research will determine whether this observation is true across all forms of PTSD-inducing stress and trauma and across all subjects.

In conclusion, it is timely to design similar evidence-based research studies to establish the strength of the evidence in support of complementary approaches for the treatment of PTSD. For example, use of complementary therapies (e.g. massage and herbal/food supplements) is widespread among active military veterans and their spouses for stress and comorbid pain and anxiety. Data indicate that up to $70 \%$ of the surveyed subjects want these interventions available at the medical treatment facility (e.g. Veterans Administration Medical Center, VAMC), despite sound supportive research data (71). This trend appears to be particularly evident among native American veterans, who usually choose not to seek treatment at VAMC facilities, in part because of the preference they hold for alternative and complementary treatments, which are usually not available at those facilities (72). This population of patients is therefore at serious risk of remaining underserved. Among the civilian population, the need for systematic

\section{Algorithm of the Process of Applying Research Evidence in Clinical Decision Making}

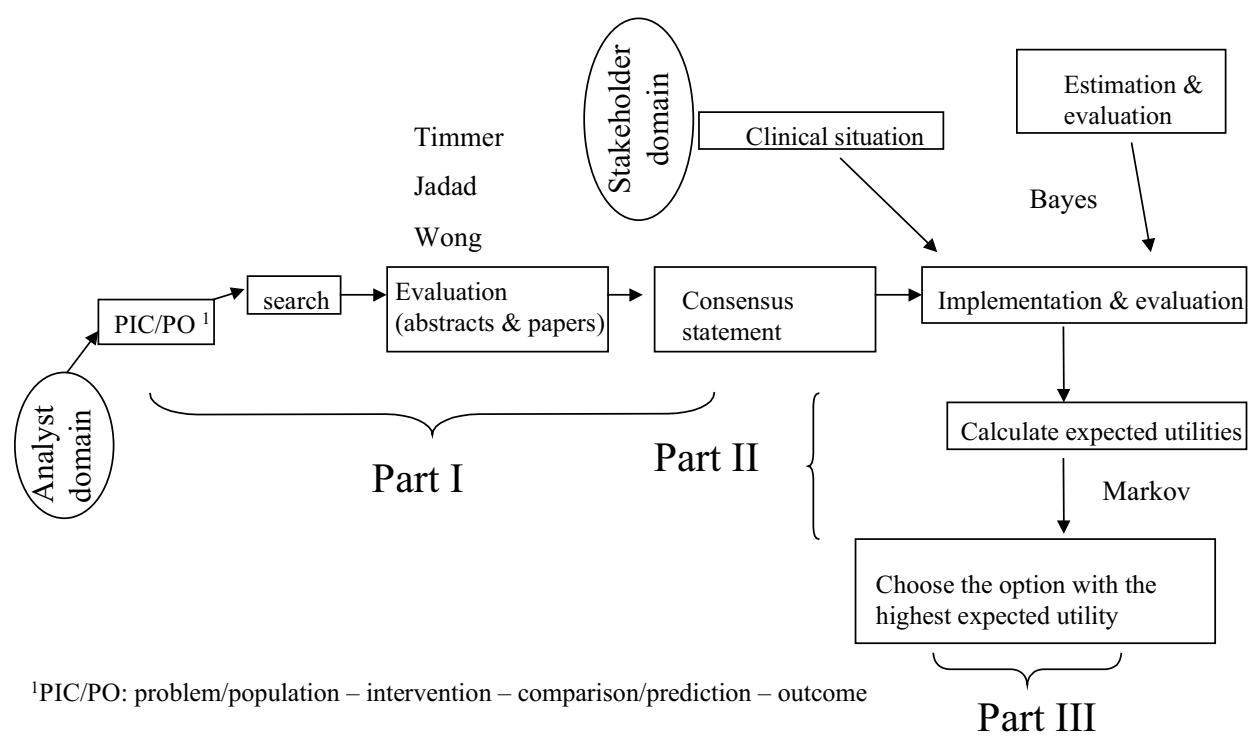

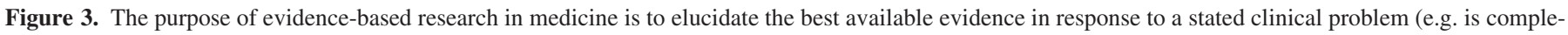

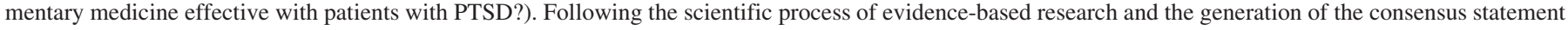

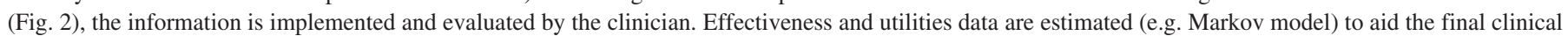
decision-making process (74). 
reviews on the benefit of complementary medicine in the treatment of PTSD is also becoming evident in light of the increasing reports proposing the benefits of massage and acupuncture in individuals exposed to the traumatic events of 9/11 (73).

Taken together, these developments should produce important novel information about the fundamental nature of PTSD from the perspective of allostasis and about its optimal treatment using the best available evidence obtained from systematic reviews. This concerted approach will be particularly important as the prevalence of PTSD with its complex psychobiological co-morbidity rises and as alternative and complementary medical treatments for PTSD emerge and take hold. Such is, in our view, the future of research in PTSD, in order to establish a registry of regular critical evaluation updates of the available evidence for the immediate service of the clinical research community and the benefit of patients with PTSD, their families and society at large.

\section{References}

1. Vojdani A, Thrasher JD. Cellular and humoral immune abnormalities in Gulf War veterans. Environ Health Perspect 2004;112:840-6.

2. McKeown-Eyssen G, Baines C, Cole DE, Riley N, Tyndale RF, et al. Case-control study of genotypes in multiple chemical sensitivity: CYP2D6, NAT1, NAT2, PON1, PON2 and MTHFR. Int J Epidemiol 2004;33:971-8.

3. Da Costa JM. On irritable heart: a clinical study of a form of functional cardiac disorder and its consequences. Am J Med Sci 1871;61:17-52.

4. Myers ABR. On the Etiology and Prevalence of Diseases of the Heart among. Soldiers. London: John Churchill \& Sons, 1870.

5. American Psychiatric Association. Diagnostic and Statistical Manual of Mental Disorders, 4th Edition. Washington, DCAmerican Psychiatric Press, 1994.

6. First MB, Spitzer RL, Gibbon M, et al. Structured Clinical Interview for DSM-IV. Washington, DC: American Psychiatric Press, 1997.

7. Lyons JA, Keane TM. Keane PTSD scale: MMPI and MMPI-2 update. J Traum Stress 1992;5:111-7.

8. Davidson JRT, Book SW, Colket JT, Tupler LA, Roth S, David D, et al. Assessment of a new self-rating scale for post-traumatic stress disorder. Psychol Med 1997;27:153-60.

9. Kubany ES, Leisen MB, Kaplan AS, Kelly MP. Validation of a brief measure of posttraumatic stress disorder: the Distressing Event Questionnaire (DEQ). Psychol Assess 2000;12:197-209.

10. Weiss DS, Marmar CR. The Impact of Event Scale-Revised. In Wilson J, Keane TM (eds). Assessing Psychological Trauma and PTSD. NY: Guilford, 1996, pp. 399-411.

11. Briere J. Psychometric review of the trauma symptom checklist-40. In: Stamm BH (ed). Measurement of Stress, Trauma, and Adaptation. Lutherville, MD: Sidran Press, 1996.

12. Blanchard EB, Jones-Alexander J, Buckley TC, Forneris CA. Psychometric properties of the PTSD Checklist (PCL). Behav Res Therap 1996;34: 669-73.

13. Norris FH, Perilla JL. The revised civilian Mississippi scale for PTSD: reliability, validity, and cross-language stability. J Traum Stress 1996;9: 285-98.

14. Foa E, Cashman L, Jaycox L, Perry K. The validation of a self-report measure of posttraumatic stress disorder: the posttraumatic diagnostic scale. Psychol Assess 1997;9:445-51.

15. King LA, King DW, Leskin GA, Foy DW. The Los Angeles symptom checklist: a self-report measure of posttraumatic stress disorder. Psychol Assess 1995;2:1-17.

16. Blake DD, Wheathers FW, Nagy LM, Kaloupeu DG, Klaumizer G, Charney DS, et al. A clinician rating scale for assessing current and lifetime PTSD: the CAPS. Behav Ther 1990;13:187-8.

17. Bremmer JD, Vermetten E, Vythilingam M, Afzal N, Schmahl C, Elzinga B, et al. Neural correlates of the classic color and emotional Stroop in women-related posttraumatic stress disorder. Biol Psychiatry 2004;55:612-20
18. Bremmer JD, Soufer R, McCarthy G, Delaney R, Staib LH, Duncan JS, et al. Gender differences in cognitive and neural correlates of remembrance of emotional words. Psychopharmacol Bull 2001;35:55-87.

19. Shin LM, Whalen PJ, Pitman RK, Bush G, Macklin ML, Lasko NB. An fMRI study of anterior cingulate function in posttraumatic stress disorder. Biol Psychiat 2001;50:932-42.

20. Keane TM, Caddell JM, Taylor KL. Mississippi scale for combat-related posttraumatic stress disorder: three studies in reliability and validity. J Consult Clin Psychol 1988;56:85-90.

21. Keane T, Wolf J, Taylor KL. Posttraumatic stress disorder: evidence for diagnostic validity and methods of psychological assessment. J Clin Psychol 1987;43:32-43.

22. Kulka RA, Schlenger WE, Fairbank JA, Hough RL, Jordan CB, Marmar CR, et al. Trauma and the Vietnam war generation: Report of findings from the National Veterans Readjustment Study. NY: Brunner/ Mazel, 1990.

23. Keane T, Fairbank J, Caddell J, Zimering R, Taylor K, Mora C. Clinical evaluation of a measure to assess combat exposure. Psychol Assess 1989;1:53-5.

24. Spitzer RL, Williams JB. Structured Clinical Interviews for DSM-III-RNon-Patient Version Modified for the Vietnam Veterans Readjustment Study. NY: New York State Psychiatric Institute, Biometrics Research, 1987.

25. Beals J, Manson SM, Shore JH, Friedman M, Ashcraft M, Fairbank JA, et al. The prevalence of posttraumatic stress disorder among American Indian Vietnam veterans: disparities and context. J Trauma Stress 2002;15:89-97.

26. Monnelly EP, Ciraulo DA, Knapp C, Keane T. Low-dose risperidone as adjunctive therapy for irritable aggression in posttraumatic stress disorder. J Clin Psychopharmacol 2003;23:193-6.

27. Barrett DH, Doebbeling CC, Schwartz DA, Voelker MD, Falter KH, Woolson RF, et al. Posttraumatic stress disorder and self-reported physical health status among US Military personnel serving during the Gulf War period: a population-based study. Psychosomatics 2002;43:195-205.

28. Conrad KJ, Wright BD, McKnight P, McFall M, Fontana A, Rosenheck R. Comparing traditional and Rasch analyses of the Mississippi PTSD scale: revealing limitations of reverse-scored items. J Appl Meas 2004;5: 15-30.

29. Elhai JD, Frueh BC, Davis JL, Jacobs GA, Hamner MB. Clinical presentations in combat veterans diagnosed with posttraumatic stress disorder. J Clin Psychol 2003;59:385-97.

30. Trent CR,Jr, Rushlau MG, Munley PH, Bloem W, Driesenga S. An ethnocultural study of posttraumatic stress disorder in AfricanAmerican and white American Vietnam War veterans. Psychol Rep 2000;87:585-9.

31. Beckham JC, Braxton LE, Kudler HS, Feldman ME, Lytle BL, Palmer S. Minnesota multiphasic personality inventory profiles of Vietnam combat veterans with posttraumatic stress disorder and their children. J Clin Psychol 1997;53:847-52.

32. Betemps EJ, Smith RM, Baker DG, Rounds-Kugler BA. Measurement precision of the clinician administered PTSD scale (CAPS): a RASCH model analysis. J Appl Meas 2003;4:59-69.

33. Engdahl B, Eberly R. Assessing PTSD among veterans exposed to war trauma 40-50 years ago. NCP Clinical Quarterly 1994;4:13-14.

34. Hovens JE, Falger PR, Op den Velde W, Schouten EG, de Groen JH, Van Duijn H. Occurrence of current post traumatic stress disorder among Dutch World War II resistance veterans according to the SCID. J Anxiety Dis 1992;6:147-57.

35. Chiappelli F, Prolo P, Cajulis E, Harper S, Sunga E, Concepcion E. Consciousness, emotional self-regulation, and the psychosomatic network: relevance to oral biology and medicine. In: Beauregard $\mathrm{M}$ (ed). Consciousness, Emotional Self-regulation and the Brain, Advances in Consciousness Research. John Benjamins Publishing Company, 2004a, 253-74.

36. Chiappelli F, Abanomy A, Hodgson D, Mazey KA, Messadi DV, Mito RS, Nishimura I, Spigleman I. Clinical, experimental and translational psychoneuroimmunology research models in oral biology and medicine. In: Ader R, Falter D and Cohen R. (eds). Psychoneuroimmunology, III. Academic Press, 2001, 645-70.

37. Chiappelli F, Cajulis OS. Psychobiological views on 'stress-related oral ulcers'. Quintess Intern 2004;35:223-7.

38. Sterling P, Eyer J. Allostasis: a new paradigm to explain arousal pathology. In: Fisher S, Reason J (eds). Handbook of Life Stress, Cognition, and Health. NY: Wiley, 1988. 
39. McEwen B, Wingfield JC. The concept of allostasis in biology and biomedicine. Hormones Behav 2003;43:2-15.

40. Schulkin J. Allostasis: a neural behavioral perspective. Hormones Behav 2003;43:21-7.

41. Foa E, Keane T, Friedman M. Effective Treatments for PTSD. NY: The Guilford Press, 2000.

42. Bradley R, Greene J, Russ E, Dutra L, Westen D. A multidimensional meta-analysis of psychotherapy for PTSD. Am J Pschiatr 2005;162: 214-27.

43. Pivac N, Kozaric-Kovacic D, Muck-Seler D. Olanzapine versus fluphenacine in an open trial in patients with psychotic combat-related posttraumatic stress disorder. Psychopharmacol 2004;175:451-6.

44. Kozaric-Kovacic D, Kocijan-Hercigonja D, Grubisic-Ilic M. Posttraumatic stress disorder and depression in soldiers with combat experience. Croat Med J 2001;42:165-70.

45. Kozaric-Kovacic D, Kocijan-Hercigonja D. Assessment of posttraumatic stress disorder and comorbidity. Mil Med 2001;166:78-83.

46. Hamner MB, Frueh C, Ulmer HG, Arana GW. Psychotic features and illness severity in combat veterans with chronic posttraumatic stress disorder. Biol Psychiat 1999;45:846-52.

47. Bisson JI, McFarlane AC, Rose S. Psychological debriefing. In: Foa E, et al. (ed). Effective Treatments for PTSD. The Guilford Press, 2000, 39-59.

48. Deahl M, Gillham A, Thomas J, Searle M, Srinivasan M. Psychological sequelae following the Gulf war: factors associated with subsequent morbidity and the effectiveness of psychological debriefing. Br J Psychiatry 1994;165:60-5.

49. Turner S, Beidel D, Frueh B. Multicomponent behavioral treatment for chronic combat-related posttraumatic stress disorder: trauma management therapy. Behavioral Mod 2005;29:39-69.

50. Sherman J. Effects of psychotherapeutic treatments for PTSD: a meta analysis of controlled clinical trials. J Trauma Stress 1998;11:413-35.

51. Van Etten ML, Taylor S. Comparative efficacy of treatments for posttraumatic stress disorder: a meta analysis. Clin Psychol Psychother 1998;5:126-44.

52. Hembree EA, Foa EB. Posttraumatic stress disorder: psychological factors and psychosocial interventions. J Clin Psychiatry 2000;61S:33-9.

53. Davidson P, Parker K. Eye movement desensitization and reprocessing (EMDR): a meta analysis. J Consult Clin Psychol 2001;69:305-16.

54. Foa E, Rothbaum B, Furr J. Augmenting exposure therapy with other CBT procedures. Psychiatr Ann 2003;33:47-53.

55. Pitman R, Orr S, Altman B, Longpre R, Poire R, Macklin M, et al. Emotional processing and outcome of imaginal flooding therapy in Vietnam veterans with chronic posttraumatic stress disorder. Compr Psychiat 1996;37:409-18.

56. Frueh BC, Turner SM, Beidel DC, Mirabella RF, Janes WJ. Trauma management therapy: a preliminary evaluation of a multicomponent behavioral treatment for chronic combat-related PTSD. Behav Res Ther 1996;34:533-43.

57. Boudewyns G, Hyer L. Physiological response to combat memories and preliminary treatment outcome in Vietnam veterans: PTSD patients treated with direct therapeutic exposure. Behav Ther 1990;21:63-87.

58. Cooper N, Clum G. Imaginal flooding as a supplementary treatment for PTSD in combat veterans: a controlled study. Beh Ther 1989;3: 381-91.
59. Hamner M, Robert S, Frueh B. Treatment resistant posttraumatic stress disorder: strategies for intervention. CNS Spectr 2004;9:740-52.

60. Stein DJ, Zungu-Dirwayi N, van Der Linden GJ, Seedat S. Pharmacotherapy for posttraumatic stress disorder. Cochrane Database Syst Rev 2000;4:CD002795.

61. Brady K, Pearlstein T, Asnis G, Baker D, Rothbaum B, Sikes C, et al. Double-blind placebo-controlled study of the efficacy and safety of sertraline treatment of posttraumatic stress disorder. J Am Med Assoc 2000;283:1837-44.

62. Davidson J, Landburg P, Pearlstein T, Weisler R, Sikes C, Farfel G. Double-blind comparison of sertraline and placebo in patients with posttraumatic stress disorder (PTSD). Abstracts of the American College of Neuropsychopharmacology 36th Annual Meeting 1997.

63. Davidson J, Malik M, Sutherland S. Response characteristics to antidepressants and placebo in post-traumatic stress disorder. Int Clin Psychopharmacol 1996;12:291-6.

64. Chung M, Min K, Jun Y, Kim S, Kim W, Jun E. Efficacy and tolerability of mirtazapine and sertraline in Korean veterans with posttraumatic stress disorder: a randomized open label trial. Hum Psychopharmacol 2004;19: 489-94.

65. Chiappelli F, Prolo P, Negoatis N, Lee A, Milkus V, Bedair D, et al. Tools and methods for evidence-based research in dental practice: preparing the future. J Evid Based Dent Pract 2004b;4:16-23.

66. Rose S, Bisson J, Wessely S. A systematic review of single-session psychological interventions ('debriefing') following trauma. Psychother Psychosom 2003;72:171-5.

67. Wessely S, Rose S, Bisson J. Brief psychological interventions ('debriefing') for trauma-related symptoms and the prevention of post traumatic stress disorder. Cochrane Database Syst Rev 2001;3: CD000560.

68. Suzanna RO, Jonathan BI, Simon WE. Psychological debriefing for preventing post traumatic stress disorder (PTSD). Cochrane Database Syst Rev 2002;2:CD000560.

69. Albucher RC, Liberzon I. Psychopharmacological treatment in PTSD: a critical review. J Psychiatr Res 2002;36:355-67.

70. Levine EG, Eckhardt J, Targ E. Change in post-traumatic stress symptoms following psychosocial treatment for breast cancer. Psychooncol 2005 Jan 13 [Epub].

71. McPherson F, Schwenka MA. Use of complementary and alternative therapies among active duty soldiers, military retirees, and family members at a military hospital. Mil Med 2004;169:354-7.

72. Westermeyer J, Canive J, Thuras $\mathrm{P}$, Chesness D, Thompson J. Perceived barriers to VA mental health care among Upper Midwest American Indian veterans: description and associations. Med Care 2002;40S: I62-71.

73. Sommers E, Porter K, DeGurski S. Providers of complementary and alternative health services in Boston respond to September 11. Am J Public Health 2002;92:1597-8.

74. Chiappelli, et al. Alzheimer's disease: new frontiers for the XXI century. In: Frank Columbus (ed). Advances in Psychology Research. Nova Science Publisher, 2005 (in press).

Received June 10, 2004; accepted September 12, 2005 


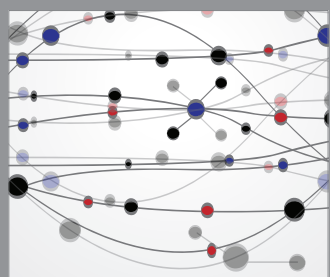

The Scientific World Journal
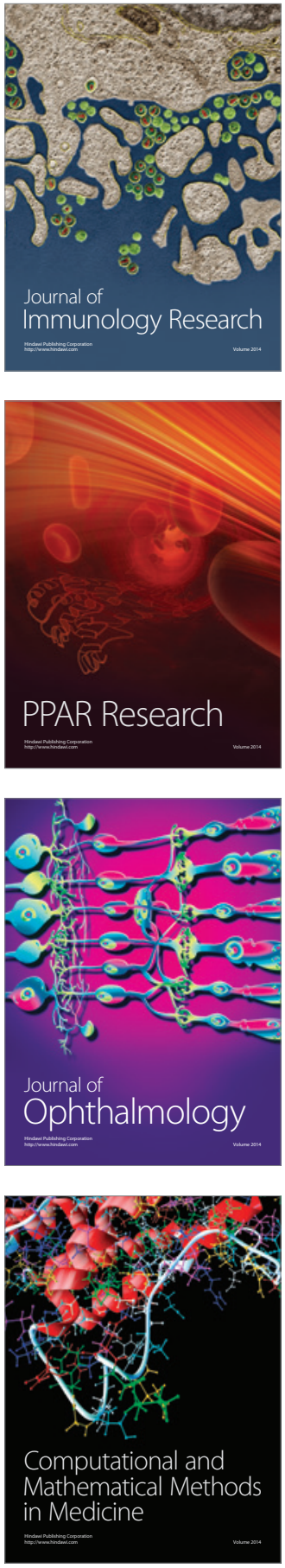

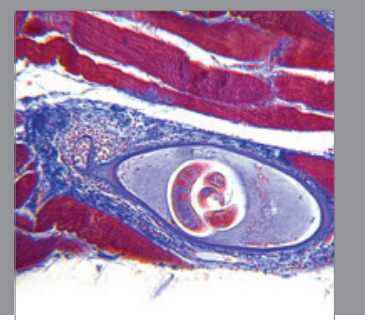

Gastroenterology

Research and Practice
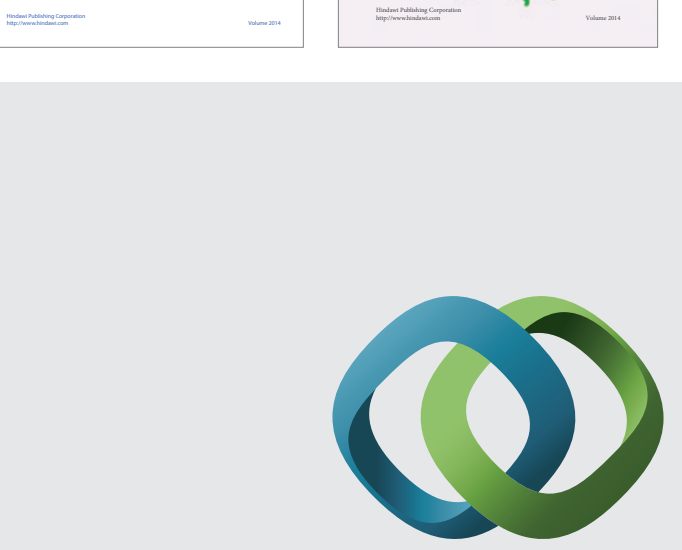

\section{Hindawi}

Submit your manuscripts at

http://www.hindawi.com
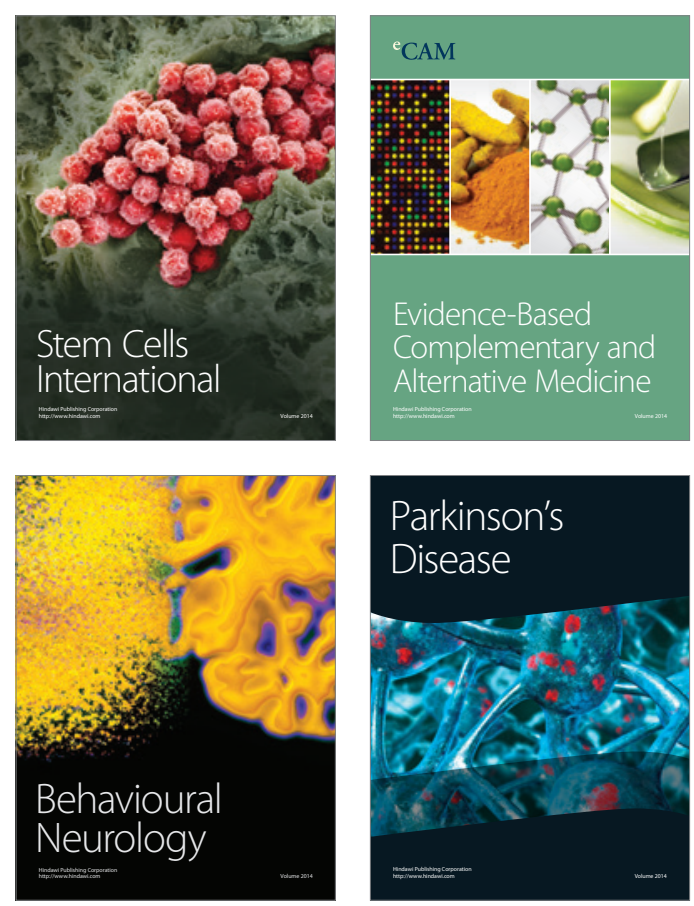

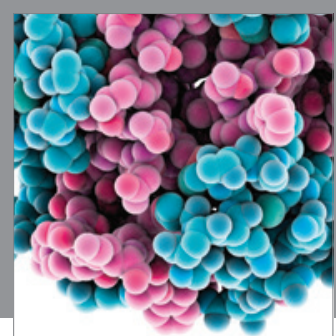

Journal of
Diabetes Research

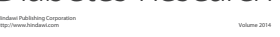

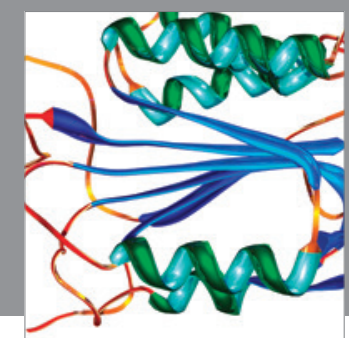

Disease Markers
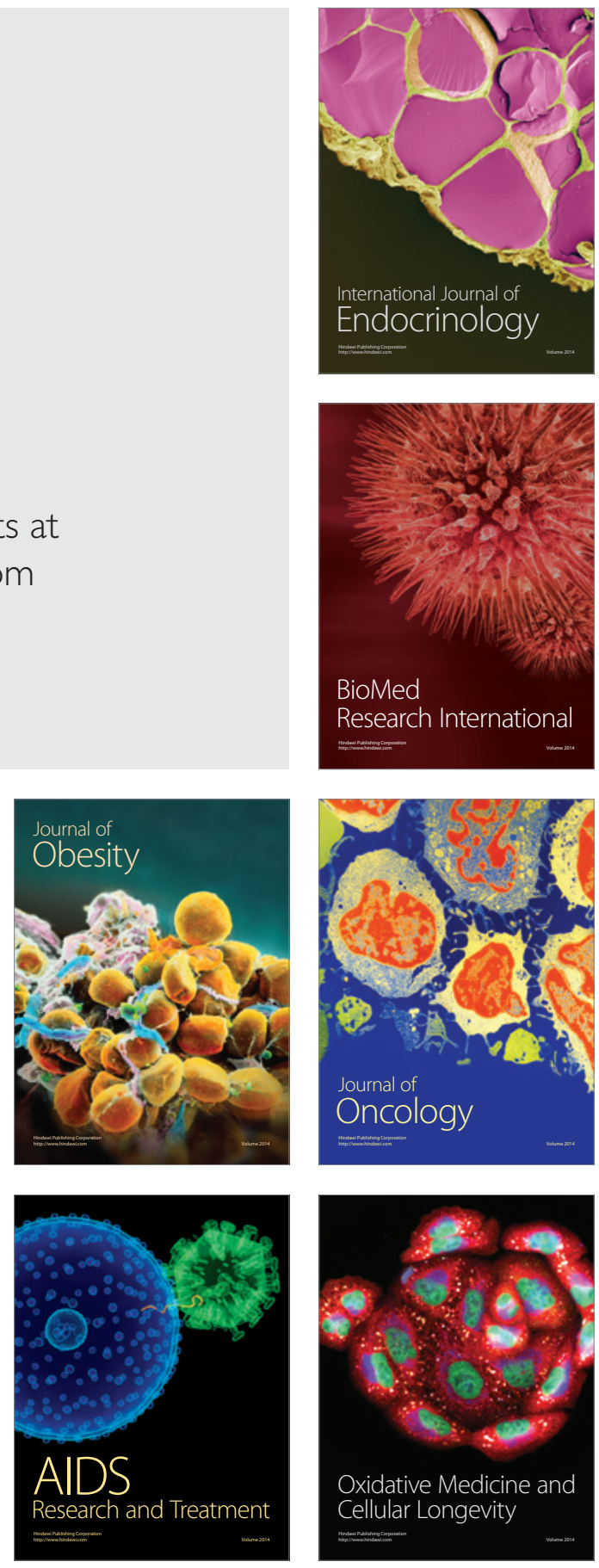